\title{
DOENÇAS DA TIREOIDE E MIASTENIA GRAVE
}

\author{
J. LAMARTINE DE ASSIS * \\ MILBERTO SCAFF ** \\ ANTONIO A. ZAMBON *** \\ PAULO E. MARCHIORI ***
}

Desde há muitos anos é conhecida e salientada a associação de endocrinopatias com a miastenia grave (MG), em particular as tireopatias 12, 15. Simpson e outros autores destacaram a associação com o hipertireoidismo 3, 16, 20, 24. A partir da hipótese de Simpson 19 de que a MG seria doença auto-imune, foram estabelecidas relações imunológicas entre $M G$ e tireopatias $1,4,7,10,16$. Numerosas pesquisas mostraram incidência relativamente alta de anticorpos anti-tireóide no soro de pacientes com $M^{2}{ }^{2}, 6,8,16,20,22,24$. Alguns autores 9,13 acenam para possivel base genética nestas associações clínicas e imunológicas.

\section{MATERIAL E METODOS}

Foram constatadas tireopatias em 15 pacientes de um grupo de 304 miastênicos (5,0\%): 10 tínham disfunção tíreoidiana e 5 exibiam bócio atóxico. As desordens funcionais da tireóide foram avaliadas mediante exames clinicos e laboratoriais. Foi pesquisado anticorpo antitireóide em 5 pacientes.

Na tabela 1 constam identificação, formas clínicas da MG, início e características da disfunção tíreoidiana, presença ou não de dados clínicos e/ou laboratoriais e influência do tratamento da disfunção endócrina na sintomatologia miastênica. A tabela 2 mostra a prevalência de tireotoxicose em pacientes da presente série comparativamente com as séries de outros autores. A tabela 3 apresenta a relação cronológica entre hipertireoidismo e MG. A tabela 4 mostra os 5 miastênicos com bócio atóxico. Nenhum dos pacientes era proveniente de zona endêmica de bócio e não havia bócio familiar.

\section{RESULTADOS}

De um grupo de 304 pacientes com MG, 15 apresentavam tireopatias (5,0\%). As idades dos pacientes variavam de 17-54 anos com a média de 33,05 anos e desvio padrão de 12,12 anos. Onze pacientes eram do sexo feminino.

No grupo com disfunção tireoidiana (Tabela 1), a forma clínica da MG era generalizada moderada em 6 pacientes ${ }_{i}$ generalizada severa em dois e generalizada acentuada em dois. Havia 9 pacientes com hipertireoidismo (3,0\%) e um com hipo-

Trabalho realizado no Serviço de Clínica Neurológica da Divisão de Neurologia do Departamento de Neuropsiquiatria (Prof. Dr. Horácio Martins Canelas) da Faculdade de Medicina da Universidade de São Paulo (FMUSP): * Professor Adjunto; ** Professor Livre Docente; *** Médico Assistente do Hospital das Clínicas, FMUSP. 
tireoidismo $(0,3 \%)$. Três pacientes tinham sinais clínicos atuais de disfunção tireoldiana (casos 3, 5 e 8); 4 tinham hipertireoidismo pregresso sendo que um foi tireoidectomizado (caso 6) e apenas um tinha hipotireoidismo (caso 4) (Tabela 1). A sintomatologia miastênica não foi influenciada pelo tratamento da disfunção tireoldiana em 5 pacientes, piorou em um e melhorou em dois; em dois outros não pôde ser avaliada (Tabela 1). A incidência da associação tireotoxicose-MG em nossa série foi significativamente menor que a referida pela maioria de outros autores (Tabela 2). A tireotoxicose apareceu primeiro em $44,0 \%$ dos casos, foi simultnkea em 33,0\%, enquanto a MG ocorreu antes em 22,0\% dos casos (Tabela 3). Os 5 pacientes com bócio não tóxico $(1,6 \%)$ eram do sexo feminino e suas idades variavam de 23-54 anos. Destes, um tinha bócio multinodular (caso 15). Em todos a função tireoidiana era normal (Tabela 4).

\begin{tabular}{|c|c|c|c|c|c|c|c|c|c|c|c|}
\hline Caso & Nome & Registro & I & $\mathbf{s}$ & C & FC & TRP & DT & $\mathbf{s c}$ & DL & TT/MG \\
\hline 1 & NM & 703186 & 40 & $\mathbf{m}$ & b & FGs & smlt & $\mathbf{H}$ & $\mathbf{A}$ & + & $\mathbf{P i}$ \\
\hline 2 & JGM & 710310 & 21 & $\mathbf{m}$ & b & FGs & após & $\mathbf{H}$ & $\mathbf{A}$ & + & In \\
\hline 3 & LC & 623039 & 44 & $\mathbf{m}$ & b & FGmd & smlt & $\mathbf{H}$ & $\mathbf{P}$ & + & In \\
\hline 4 & CMS & & 17 & $f$ & b & FGmd & smlt & HO & $\mathbf{A}$ & + & $\mathbf{M}$ \\
\hline 5 & MNG & & 31 & $\mathbf{f}$ & b & FGmd & smlt & $\mathbf{H}$ & $\mathbf{P}$ & + & In \\
\hline 6 & OSPC & 21254101 & 27 & $\mathbf{f}$ & b & $\mathbf{F G a}$ & antes & $\mathbf{H}$ & $P^{*}$ & + & $\mathbf{N}$ \\
\hline 7 & $\mathbf{R C}$ & 2146732F & 31 & $\mathbf{f}$ & b & FGmd & após & $\mathbf{H}$ & $P^{* *}$ & + & In \\
\hline 8 & PAT & 2201111A & 24 & $\mathbf{m}$ & p & FGmd & antes & $\mathbf{H}$ & $\mathbf{P}$ & + & $\mathbf{M}$ \\
\hline 9 & $\mathbf{M P}$ & 2108601 & 28 & $\mathbf{f}$ & $\mathbf{b}$ & $\mathbf{F G a}$ & antes & $\mathbf{H}$ & $P^{* *}$ & + & $\mathbf{N}$ \\
\hline 10 & MMPT & $2165379 F$ & 47 & $\mathbf{f}$ & b & FGmd & antes & $\mathbf{H}$ & $\mathbf{P}^{* *}$ & $t$ & In \\
\hline
\end{tabular}

Tabela 1 - Disfunção tireoidiana (DT) em 304 pacientes com miastenia grave (MG): $I$, idade em anos; $S$, sexo ( $m$, masculino; $f$, feminino); $C$, cor (b, branca; $p$, pardia); FC forma clinica (FG, generalizada: s, severa; md, moderada; a, acentuada); TRP, inicio da tireopatia: simultaneo (smlt), após ou antes o da MG; $D T$, disfunço tireoidiana ( $H$, hipertireoidismo; HO, hipotireoidismo); $S C$, sinais clinicios ( $A$, ausemtes; $P$, presentes; $P^{*}$, hipertireoidismo pregresso $e$ tireoidectomia; $P^{* *}$, hipertireoidismo pregresso); $D L$, dados laboratoriais ( + , positivos); $D T / M G$, influencia do tratamento da $D T$ na $M G$ (Pi, piorado; In, inalterado; $M$, melhorado; $N$, não avaliado).

\begin{tabular}{lcccc}
\hline Autores & Ano & $\begin{array}{c}\text { Pacientes } \\
\text { (número) }\end{array}$ & $\begin{array}{c}\text { Tireotoxicose } \\
\text { (número) }\end{array}$ & $\%$ \\
\hline Oosterhuis & 1964 & 164 & 8 & 4,8 \\
Wolf \& col. & 1966 & 399 & 22 & 5,5 \\
Osserman \& col. & 1967 & 801 & 42 & $\mathbf{5 , 2}$ \\
Simpson \& col. & 1966 & 295 & 24 & $\mathbf{8 , 1}$ \\
Assis \& col. & 1984 & 304 & 9 & $\mathbf{3 , 0}$ \\
Total & & 1963 & 105 & 5,3 \\
\hline
\end{tabular}

Tabela 2-Disfunç̃o tireoidiana e miastenia grave. Prevalencia da tireotoxicose em pacientes com miastenia grave. 


\begin{tabular}{lccr}
\hline Aspectos analisados & $\begin{array}{c}\text { Millikan e Haines } \\
(\mathbf{1 9 5 3 )}\end{array}$ & $\begin{array}{c}\text { Rowland } \\
(\mathbf{1 9 7 7 )}\end{array}$ & $\begin{array}{c}\text { Assis e col. } \\
(1984)\end{array}$ \\
\hline \hline Número de pacientes & 25 & 20 & 9 \\
Tireotoxicose primeiro (\%) & 48 & 50 & 44 \\
Inicio simultâneo (\%) & 20 & 30 & 33 \\
Miastenia grave primeiro (\%) & 32 & 20 & 22 \\
\hline
\end{tabular}

Tabela 3 - Tireotoxicose e miastenia grave: relaçáo cronologica entre miastenia grave e tireotoxicose: estudo comparado com séries de outros autores.

\begin{tabular}{|c|c|c|c|c|c|}
\hline \multirow[b]{2}{*}{ Caso } & \multirow[b]{2}{*}{11} & \multirow[b]{2}{*}{12} & \multicolumn{2}{|c|}{ Bócio } & \multirow[t]{2}{*}{ ico } \\
\hline & & & 13 & 14 & \\
\hline Nome & MB & AK & BFO & $\mathbf{A J}$ & IBM \\
\hline Idade & 36 & 37 & 39 & 23 & 54 \\
\hline Sexo & $\mathbf{F}$ & $\mathbf{F}$ & $\mathbf{F}$ & $\mathbf{F}$ & $\mathbf{F}$ \\
\hline Captação de $2 \mathrm{~h}$ & 13,0 & 9,5 & 15,0 & 23,0 & \\
\hline Iodo radioativo (\% $24 \mathrm{~h}$ ) & 29,0 & 45,5 & 33,0 & 58,0 & \\
\hline Iodemia proteica (BI 127) $\mathrm{mcg} / 100 \mathrm{ml}$ & 6,8 & 3,8 & 4,9 & 6,5 & \\
\hline M. basal (\%) & +15 & -1 & +12 & +23 & \\
\hline Colesterol $(\mathrm{mg} / 100 \mathrm{ml})$ & 249 & 190 & 147 & 234 & \\
\hline Anticorpo anti-tireóide & neg. & neg. & neg. & neg. & \\
\hline Bócio presente & dif. & dif. & dif. & dif. & $\mathbf{m}-\mathbf{n}$ \\
\hline \multirow[t]{2}{*}{ Observação } & & & & $\mathbf{T} 3=\mathbf{N}$ & $\mathbf{T} 3=\mathbf{N}$ \\
\hline & & & & & $\mathbf{T} 4=\mathbf{N}$ \\
\hline
\end{tabular}

Tabela 4 - Bócio não toxxico em 304 pacientes miastenicos; caso (número), nome, idade (em anos), sexo ( $F$, femlinino); $N$, mormal; neg., negativo; dif, difuso; $m-n$, multiwodular; TS, dosagem da tri-ioda-tironina em ng por ml mediante radimunensaio; T4, tiroxina plasmatica medida por radimunensaio em mog por $100 \mathrm{ml}$.

\section{COMENTARIOS}

Em um grupo de 304 pacientes com MG as tireopatias constituíram, após os timomas $(5,5 \%)$, as doenças mais frequentemente associadas com MG $(5,0 \%)$. Ocorreu predomínio acentuado da tireotoxicose $(3,0 \%)$ sobre o hipotireoidismo $(0,3 \%)$, o que está de acordo com a literatura $16,19,20,24$. No entanto, a proporção de tireotoxicose nesse grupo de miastênicos é inferior à maioria daquela referida por outros autores $14,16,21,24$ (Tabela 2). A miastenia precedeu o aparecimento da tireotoxicose em 22,0\% dos casos, o que se aproxima dos achados de outros autores 12, 18. Na nossa série a tireotoxicose precedeu em 44,0\% dos casos a MG, o que também está bem próximo dos números referidos na lite- 
ratura 12, 18, o mesmo acontecendo quanto a simultaneidade do aparecimento das duas doenças 12, 18 (Tabela 3). São pouco relevantes no binômio MG-tireotoxicose, os aspectos clínicos da tireopatia e a repercussão terapêutica da disfunção tireoidiana na sintomatologia miastênica. Os 5 pacientes sem sinais clínicos atuais de hipertireoidismo exibiam FGM, FGA ou FGS. Os três pacientes com sinais clínicos atuais de tireotoxicose tinham FGM de MG. $O$ único paciente com hipotireoidismo (caso 4) tinha FGM. Estes achados sugerem não haver correlação entre intensidade da sintomatologia miastênica e da disfunção tireoidiana. Deste modo, um paciente com tireotoxicose clínica e laboratorial acentuada pode evoluir com sintomatologia miastênica moderada e vice-versa.

$\mathrm{Na}$ série atual, na maioria das vezes, não houve correlação entre remissão terapêutica do hipertireoidismo e melhora clínica da MG: em três pacientes a normalizaçãao do hipertireoidismo não influenciou a evolução dos sintomas miastênicos e em três outros com tireotoxicose pregressa, do mesmo modo,a normalização da função tireoidiana não modificou a evolução da MG. É de notar que em um destes pacientes (caso 10) a tireotoxicose precedeu a MG e a normalização da função tireodiana não impediu a eclosão ulterior da moléstia. Ocorreu melhora da MG com a normalização terapêutica da função tireoidiana em apenas 2 pacientes: um com hipotireoidismo (caso 4) e outro com hipertireoidismo (caso 8). Um paciente piorou (caso 1) e dois outros não puderam ser acompanhados (casos 6 e 9). No nosso grupo a evidência clínica e/ou laboratorial de tireoidite não ocorreu. Apenas um paciente (caso 4), com hipotireoidismo, teve provável episódio de tireoidite, que cedeu com o tratamento. Durante o episódio a sintomatologia miastênica não foi influenciada, porém com a normalização da função tiresidiana houve melhora de sintomatologia miastênica. A raridade da associação de $M G$ com doença de Hashimoto é referida na literatura $5,17,21$. No entanto, alguns autores ${ }^{1}$ presumem que diversos pacientes com MG e tireoidite tenham função tireoidiana normal ou ligeiramente alterada e, nestas condições, a tireopatia não seria diagnosticada a não ser mediante exame histológico.

\section{RESUMO}

Os autores estudam o comportamento da tireóide em um grupo de 304 pacientes com miastenia grave, constatando 15 casos de tireopatias, sendo 9 de hipertireoidismo, um de hipotireoidismo e 5 de bócio sem alterações da função tireoidiana. Nenhum paciente era proveniente de região de bócio endêmico e não havia bócio familiar. A tireotoxicose, que foi a disfunção mais frequente, foi estudada quanto a sua prevalência em pacientes miastênicos, quanto a sua influência sobre os sintomas da miastenia grave e quanto à época do seu aparecimento. 


\section{SUMMARY}

Thyroid diseases and myasthenia gravis: study of 15 cases.

In a group of 304 myasthenic patients 15 cases with thyropathies were reported: nine with hiperthyroidism, one with hypothyroidism and five with nontoxic goiter. Four patients presented diffuse simple goiter and one a multinodular goiter with normal thyroid function. No patient came from an endemic goiter region, not even familial goiter. The prevalence and influence of hyperthyroidism on myasthenic symptomatology were studied. Our findings suggest that there is no clinical correlation between both myasthenia symptomatology and thyroid dysfunction, neither significant influence on myasthenic symptoms when the endocrine disorders improve.

\section{REFERPNCIAS}

1. AARLI, J. A.; THUNOLD, S. \& HEIMANN, P. - Thyroiditis in myasthenia gravis. Acta neurol. scand. 58:121, 1978.

2. ADNER, M. M.; SHERMAN, J. D.; ISE, C.; SCHWAB, R. S. \& DAMESHEK, W. - An immunologic survey of 48 patients with myasthenia gravis. New Engl. J. Med. 271:1327, 1964.

3. ASSIS, J. L. - Miastenia grave. In R. Melarangno \& C. K. Naspitz (eds.) Neuroimunologia. Sarvier, São Paulo, 1982, pg 97.

4. BECKER, K. L.; TITUS, J. L.; MCCONAHEY, W. M. \& WOOLNER, L. B. Morphologic evidence of thyroiditis in myasthenia gravis. J. amer. med. Assoc. 18:994, 1964.

5. DALY, J. J. \& JACKSON, E. - Case of Hashimoto disease with myasthenia gravis. Brit. med. J. 1:748, 1964.

6. DOWNES, J. M.; GREENWOD, B. M. \& WRAY, S. H. - Autoimmune aspects of myasthenia. Quart. J. Med. 35:85, 1966.

7. DRACHMAN, D. B. - Myasthenia gravis and thyroid gland. New Engl. J. Med. 266:330, 1962.

8. FELTKAMP, T. E. W.; VAN DEN BERG-LOONEN, P. M.; NIJENHIUS, L. E.; ENGELFRIET, C. P.; VAN ROSSUM, A. L.; VAN LOGHEM, J. J. \& OOSTERHUIS, H. J. G. H. -Myasthenia gravis, autoantibodies and HLA antigens. Brit. med. J. 1:131, 1974 .

9. GELTHORPE H. K.; MILFORD-WARD, A. \& DAVIES-JONES, G. - Clinical and immunological associations in myasthenia gravis: autoantibodies. J. Neurol. Neurosurg. Psychiat. 43:967, 1980.

10. KIESSliNG, W. R.; PFLUGhaUPT, K. W.; RICKER, K.; HAUHITZ, I. \& MERTENS, H. G. - Thyroid function and circulating antithyroid antibodies in myasthenia gravis. Neurology (Ny) 31:771, 1981.

11. MEDEIROS NETO, G. A.; ASSIS, J.L.; PIERONI, R. R. \& NICOLAU, W. Miastenia grave e função tireoidiana. Rev. Ass. méd. bras. 10:265, 1964.

12. MILLIKAN, C. H. \& HAINES, S. F. - The thyroid gland in relation to neuromuscular disease. Arch. int. Med. 92:5, 1953.

13. NAMBA, T. \& GROB, D. - Myasthenia gravis and hyperthyroidism occurring in two sisters. Neurology (Minneapolis) 21:377, 1971.

14. OOSTERHUIS, H. J. G. H. - Studies in myasthenia gravis: a clinical study of 180 patients. J. neurol. Sci. 1:1546, 1964.

15. OSSERMAN, K. E. - Myasthenia Gravis. Grune \& Stratton, New York, 1958.

16. OSSERMAN, K. E.; TSAIRIS, P. \& WEINER, L. B. - Myasthenia gravis and thyroid disease: clinical and immunologic correlation. J. Mt. Sinai Hosp. 34:469, 1967. 
17. RAMSAY, I. - Thyroid Disease and Muscle Dysfunction. Heinemann, London, 1974, pg. 53.

18. ROWLAND, L. P. - Myasthenia Gravis. In E. S. Goldensohn \& S. H., Appel (eds.) - Scientific Approaches to Clinical Neurology. Lea \& Febiger, Philadelphia, 1977, pg 1018.

19. SIMPSON, J. A. - Myasthenia gravis: a new hypothesis. Scott. med. J. 5:419, 1960.

20. SIMPSON, J. A. - Myasthenia gravis as an autoimmune disease: clinical aspects. Ann. N. Y. Acad. Sci. 135:506, 1966.

21. SIMPSON, J. F.; WESTENBERG, M. R. \& MAGEE, H. R. - Myasthenia gravis: an analysis of 295 cases. Acta neurol. scand. 42 (suppl. 23):7, 1966.

22. VAN DER GELD, H.; FELTKAMP, T. E. W.; VAN DER LOGHEM, J. J.; OOSTERHUIS H. J. G. H. \& BIEMOND, A. - Multiple antibody production in myasthenia gravis. Lancet $2: 373,1963$.

23. WILLIAMSON, H. O.; PHANSEY, S. A.; MATHUR, S.; MATHUR, R. S.; BAKER, F. R. \& FUDFNBERG, H. H. - Myasthenia gravis, premature menopause, and thyroid autoimmunity. Amer. J. Obstet. Gynec. 137:893, 1980.

24. WOLF, S. M.; ROWLAND, L. P.; SCHOTLAND, D. L.; McKINNEY, A. S.; HOFFFER, P. F. A. \& ARANOW, Jr., H. - Myasthenia as an auto-immune disease: clinical aspects. Ann. N. Y. Acad. Sci. 135:517, 1966.

Clinica Neurolbgica, FMUSP - Cahica Postal 3461 - 01000, sdo Paulo, SP - Brasil 